\title{
Carbono e frações granulométricas da matéria orgânica do solo sob sistemas de produção orgânica
}

\author{
Carbon and granulometry fractions of soil organic matter under organic production system
}

\author{
Arcângelo Loss ${ }^{I *}$ Marcos Gervasio Pereira ${ }^{I}$ Nivaldo Schultz ${ }^{I}$ Lúcia Helena Cunha dos Anjos ${ }^{I}$ \\ Eliane Maria Ribeiro da Silva ${ }^{I I}$
}

\section{RESUMO}

Os resultados do fracionamento granulométrico da matéria orgânica do solo (MOS) podem contribuir para o entendimento da sua dinâmica em áreas com sistemas de manejo orgânico. O objetivo deste trabalho foi avaliar as frações granulométricas da MOS e o carbono orgânico total em áreas submetidas a manejo orgânico sob diferentes sistemas de uso do solo. As áreas selecionadas apresentavam os seguintes sistemas: preparo convencional (PC, milho/feijão), plantio direto ( $P D$, beringela/milho), consórcio maracujá - Desmodium sp, uma área cultivada com figo e um sistema agroflorestal (SAF). As amostras de solo foram coletadas em duas profundidades (0-5 e 5-10cm) e duas épocas (verão - 17 de novembro de 2005 e inverno - 23 de junho de 2006-). Foi medido o carbono orgânico total (COT), calculado seu estoque e realizado o fracionamento granulométrico da MOS, obtendose o carbono orgânico particulado (COp) e o carbono orgânico associado aos minerais (COam). As áreas com figo e beringela/ milho apresentaram os maiores teores de COT e estoques de COT na estação do verão. No inverno, os sistemas com rotação de culturas (PD e PC) foram mais eficientes no estoque de COT que os demais sistemas avaliados. Maiores variações foram observadas nos teores de COT na profundidade de $5-10 \mathrm{~cm}$, decorrentes dos sistemas de manejo adotados. Pelos valores de COp foi possível identificar diferenças entre todos os sistemas de uso avaliados na estação do verão e na profundidade de 0 $5 \mathrm{~cm}$, destacando-se a área em plantio direto com os maiores valores, nas duas estações. O COp mostrou-se mais eficiente que o COT no verão para evidenciar diferenças entre os sistemas avaliados na profundidade de $0-5 \mathrm{~cm}$. As diferenças observadas entre os sistemas de manejo para o COam foram atribuídas ao uso constante da adubação orgânica.

\begin{abstract}
The soil organic matter (SOM) granulometry fractioning can contribute to the understanding of the SOM dynamics in areas under organic production systems. The objective of this study was to evaluate the granulometry fractions of SOM and total organic carbon in areas with organic management systems and under different soil usages. The selected areas presented the following systems: conventional tillage (CT, corn/beans), no tillage (NT, eggplant/corn), passion fruit and Desmodium sp consortium, fig grove, and agroforest system (AFS). Soil samples were taken in two depths (0-5 and $5-10 \mathrm{~cm})$ and two periods of the year (17/11/2005 - summer and 23/06/2006 - winter). The total organic carbon (TOC) was evaluated, and calculated the TOC stocks. The SOM was granulometrically fractioned, obtaining the particulate organic carbon (POC) and organic carbon associated to minerals (OCam). The fig grove and eggplant/corn areas presented the highest TOC and TOC stocks in the summer season. In the winter the crop rotation (NT and CT) systems were more efficient in the stocking of TOC that the other systems. There were observed highest TOC variations in the $5-10 \mathrm{~cm}$ depth, resulting from the management systems adopted. From the POC values it was possible to identify differences among all systems evaluated, in the summer season and in the $0-5 \mathrm{~cm}$ depth, standing out the area with no tillage, which showed the highest values, in the two seasons. The POC was more efficient than the TOC, in the summer, to evidence differences among the evaluated soil usage systems, in the $0-5 \mathrm{~cm}$ depth. The differences observed among the production systems for OCam were attributed to the constant usage of organic manure.
\end{abstract}

Key words: organic manuring, crop residues, particulate organic matter.
Palavras-chave: adubação orgânica, resíduos vegetais, matéria orgânica particulada.

IDepartamento de Solos (DS), Universidade Federal Rural do Rio de Janeiro (UFRRJ), 23890-000, Seropédica, RJ, Brasil. Email:arcangeloloss@yahoo.com.br.*Autor para correspondência.

IICentro Nacional de Pesquisa em Agrobiologia (CNPAB), Embrapa Agrobiologia, Seropédica, RJ, Brasil. 


\section{INTRODUÇÃO}

A preocupação com a sustentabilidade agrícola é crescente e tem estado em evidência nos últimos anos. Assim, a qualidade do solo é um dos fatores-chave para se atingir a sustentabilidade de um sistema de produção, destacando-se o manejo empregado como o componente principal para se obter uma produção sustentável. Partindo-se desse pressuposto, o manejo orgânico pode ser uma forma adequada de se alcançar um sistema agrícola sustentável. Entretanto, existem poucos estudos em áreas com esse tipo de manejo no Brasil referentes aos efeitos causados às frações orgânicas da matéria orgânica do solo (MOS), provenientes desse manejo sob culturas de frutíferas e oleráceas.

Nesse sentido, o fracionamento granulométrico da MOS em seus compartimentos pode auxiliar na avaliação das modificações decorrentes do uso devido à maior sensibilidade dessas frações frente ao manejo (CAMBARDELLA \& ELLIOTT, 1992; BAYER et al., 2004; NICOLOSO, 2005). A utilização de sistemas de manejo que promovam diferentes aportes de biomassa vegetal pode ser identificada por meio da fração particulada da MOS, sendo possível esta ser utilizada como ferramenta para avaliar a qualidade do solo, principalmente em um curto período de tempo (CONCEIÇÃO et al., 2005).

O fracionamento granulométrico da MOS (CAMBARDELLA \& ELLIOTT, 1992) consiste na separação de duas frações orgânicas: o carbono orgânico particulado (COp) e o carbono orgânico associado aos minerais (COam). O COp é a fração da MOS separada por dispersão e peneiramento do solo associada à fração areia $(\mathrm{COp}>53 \mu \mathrm{m})$, sendo caracterizado como partículas derivadas de resíduos de plantas e hifas com estrutura celulares reconhecíveis, cuja permanência no solo está condicionada à proteção física desempenhada por agregados (GOLCHIN et al., 1994a). O COam é a fração da MOS associada às frações silte e argila do solo (COam $<53 \mu \mathrm{m})$, sendo definida como a fração da MOS que interage com a superfície de partículas minerais, formando os complexos organominerais, estando protegida pelo mecanismo de proteção coloidal (CHRISTENSEN, 1996b).

O manejo orgânico pode influenciar positivamente os atributos químicos e físicos do solo. Avaliando as propriedades edáficas em áreas sob manejo orgânico em Argissolo Vermelho-Amarelo em Nova Friburgo, Rio de Janeiro(RJ), CARDOZO etal. (2008) observaram efeito positivo do manejo orgânico nas propriedades físicas (densidade do solo e agregação) e químicas $(\mathrm{pH}, \mathrm{Ca}, \mathrm{Mg}, \mathrm{K}, \mathrm{P})$, apresentando maiores valores desses atributos quando comparados à área de floresta secundária, principalmente na camada de 0 $5 \mathrm{~cm}$.

O objetivo deste trabalho foi avaliar as frações granulométricas da MOS e o carbono orgânico em áreas submetidas a manejo orgânico sob diferentes sistemas de uso do solo.

\section{MATERIAL E MÉTODOS}

O estudo foi realizado em área do Sistema Integrado de Produção Agroecológica - SIPA, que foi implantado em 1993 e compreende uma área de 59ha destinada ao exercício da agroecologia. O SIPA está localizado na Embrapa Agrobiologia, Seropédica, RJ, situado na latitude $22^{\circ} 45^{\prime} \mathrm{S}$, longitude $43^{\circ} 41^{\prime} \mathrm{W}$ Grw e altitude de $33 \mathrm{~m}$, sendo o clima incluído na classificação de Köppen como do tipo Aw. O solo da área experimental foi classificado como Argissolo Vermelho-Amarelo (EMBRAPA, 2006), com textura franco arenosa no horizonte $A$, sendo rotineiramente cultivado com oleráceas e frutíferas.

Foram selecionadas cinco áreas de 0,12ha cada, sendo estas: cultivo de figo (Ficus carica) com sete anos e as entrelinhas com gramíneas (Paspalum notatum); consórcio maracujá (Passiflora edulis) Desmodium sp, sendo essa área cultivada com maracujá desde 1996; cultivo de milho (Zea mays) no verão e feijão (Phaseolus vulgaris) no inverno, em área onde há oito anos vêm sendo conduzidos experimentos com rotação de culturas, tais como milho (Zea mays), feijão (Phaseolus vulgaris), quiabo (Abelmoschus esculentus), couve (Brassica oleracea), beringela (Sonalum melogena), e com revolvimento do solo (aração e gradagem); cultivo de beringela no verão e milho no inverno, com a mesma seqüência de rotação de culturas e tempo de utilização da área de milho/ feijão, entretanto em plantio direto; e sistema agroflorestal (SAF) com cinco anos de implantação, sendo formado por banana (Musa sapientum), palmito jussara (Euterpe oleracea), cacau (Thebroma cacao), mamão (Carica papaya), guapuruvu (Schizolobium parahyba), dentre outras.

O manejo e a adubação das áreas avaliadas para este estudo foram realizados da seguinte forma: 0 SAF não recebeu nenhum tipo de adubação complementar, ou seja, o fornecimento de nutrientes é decorrente do aporte e da decomposição do material vegetal das espécies presentes no sistema. A área sob cobertura de maracujá foi adubada nas covas com esterco bovino no momento do plantio, recebendo duas adubações de cobertura com "cama" de aviário por ano. As áreas com as coberturas vegetais de figo, milho/ 
feijão e beringela/milho receberam adubação com "cama" de aviário no momento do plantio e depois em cobertura, de acordo com as necessidades de cada cultura, sendo o equivalente à dose de $100 \mathrm{~kg} \mathrm{ha}^{-1} \mathrm{de} \mathrm{N}$.

$\mathrm{Na}$ área com plantio de figo, no período anterior ao deste estudo (de 1999 - início do plantio até o ano de 2002), havia sido realizado consórcio da frutífera com crotalária (Crotalaria juncea) e siratro (Macroptilium artropurpureum). Na época do estudo, no entanto, não havia nenhuma cultura, sendo somente verificada na área a presença de cobertura morta, composta por resíduos vegetais provenientes do corte da grama batatais (Paspalum notatum), o que é mais largamente utilizado no verão.

Nas áreas de milho/feijão e beringela/milho, no período anterior à instalação dessas culturas, eram plantadas mucuna cinza (Mucuna pruriens), mucuna anã (Mucuna deeringiana), Crotalaria spectabilis e Crotalaria juncea, consorciadas com a cultura principal. Entretanto, no momento das coletas essas leguminosas não estavam presentes na área.

A coleta das amostras de solo foi realizada em duas ocasiões: a primeira, no dia 17 de novembro de 2005 (estação do verão); e a segunda, no dia 23 de junho de 2006 (estação do inverno). Em cada uma das áreas foram coletadas cinco amostras compostas formadas por três amostras simples. Estas foram coletadas entre as linhas de cada cultura, com auxílio de enxadão e espátula, nas profundidades de $0-5$ e $5-10 \mathrm{~cm}$. O material foi seco ao ar e peneirado em malha de 2,00mm para realização das análises. Foram avaliados o carbono orgânico total (COT), segundo EMBRAPA (1997), e o fracionamento granulométrico da MOS (CAMBARDELLA \& ELLIOTT, 1992), obtendo-se o carbono orgânico particulado (COp) e carbono orgânico associado aos minerais (COam).

Aproximadamente $20 \mathrm{~g}$ de solo e $60 \mathrm{~mL}$ de solução de hexametafosfato de sódio $\left(5 \mathrm{~g} \mathrm{~L}^{-1}\right)$ foram agitados durante 15 horas em agitador horizontal. A seguir, a suspensão foi passada por peneira de $53 \mu \mathrm{m}$. $\mathrm{O}$ material retido na peneira (COp) foi seco em estufa a $50^{\circ} \mathrm{C}$, quantificado em relação a sua massa, moído em gral de porcelana e analisado em relação ao teor de COT (EMBRAPA, 1997). O COam foi obtido a partir da diferença ente COT e COp.

O delineamento utilizado foi o inteiramente casualizado, sendo realizadas análises estatísticas entre as áreas e entre as estações, ambas com cinco repetições. Para os dados obtidos, em cada profundidade, foi feita avaliação da normalidade dos dados e homogeneidade das variâncias dos erros. Posteriormente, os resultados foram submetidos à análise de variância com aplicação do teste $\mathrm{F}$, e os valores médios comparados entre si pelo teste de ScottKnott a 5\%.

\section{RESULTADOS E DISCUSSÃO}

Os teores de COT tenderam a ser mais elevados na camada de $0 \mathrm{a} 5 \mathrm{~cm}$ em relação à camada de $5-10 \mathrm{~cm}$, com exceção da área de milho/feijão, para as duas estações avaliadas (Tabela 1). Esse comportamento demonstra a maior influência no teor de MOS dos resíduos vegetais deixados em superfície pelas diferentes coberturas vegetais analisadas. $\mathrm{Na}$ área de milho/feijão, o maior teor de COT encontrado na estação do inverno, profundidade de $5-10 \mathrm{~cm}$, provavelmente foi devido ao preparo do solo, pois antes da coleta das amostras a área foi arada e gradeada, sendo os resíduos culturais do milho incorporados e homogeneizados nos primeiros $10 \mathrm{~cm}$ de solo para plantio da cultura do feijão.

As áreas de figo e beringela/milho apresentaram teores de COT no verão maiores que as demais, nas duas profundidades, destacando-se a área

Tabela 1 - Concentração de carbono orgânico total (COT) e estoque de COT em função dos diferentes sistemas de uso do solo.

\begin{tabular}{|c|c|c|}
\hline & Verão & Inverno \\
\hline Sistemas de uso do solo & \multicolumn{2}{|c|}{$\operatorname{COT}\left(\mathrm{g} \mathrm{kg}^{-1}\right)$} \\
\hline Beringela/Milho (PD) & $10,9 \mathrm{~B}^{\mathrm{ns}}$ & $9,8 \mathrm{~A}^{\mathrm{ns}}$ \\
\hline Milho/Feijão (PC) & $9,5 \mathrm{C}^{\mathrm{ns}}$ & $9,1 \mathrm{~B}^{\mathrm{ns}}$ \\
\hline Figo & $13,7 \mathrm{Aa}$ & $9,1 \mathrm{Bb}$ \\
\hline Maracujá & $9,1 \mathrm{C}^{\mathrm{ns}}$ & $8,8 \mathrm{~B}^{\mathrm{ns}}$ \\
\hline \multirow[t]{2}{*}{ SAF } & $9,4 C^{\text {ns }}$ & $9,0 \mathrm{~B}^{\mathrm{ns}}$ \\
\hline & \multicolumn{2}{|c|}{ 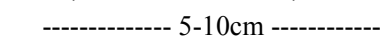 } \\
\hline Beringela/Milho (PD) & $9,5 \mathrm{~B}^{\mathrm{ns}}$ & $9,3 \mathrm{~B}^{\mathrm{ns}}$ \\
\hline Milho/Feijão (PC) & $8,9 \mathrm{Cb}$ & $10,3 \mathrm{Aa}$ \\
\hline Figo & $11,5 \mathrm{Aa}$ & $7,8 \mathrm{Cb}$ \\
\hline Maracujá & $7,4 \mathrm{Db}$ & $8,2 \mathrm{Ca}$ \\
\hline \multirow[t]{3}{*}{ SAF } & $7,0 \mathrm{~Eb}$ & $8,1 \mathrm{Ca}$ \\
\hline & \multicolumn{2}{|c|}{ Estoque de COT $\left(\mathrm{Mg} \mathrm{ha}^{-1}\right)$} \\
\hline & ----------- & ----------- \\
\hline Beringela/Milho (PD) & $7,1 \mathrm{~B}^{\mathrm{ns}}$ & $7,1 \mathrm{~A}^{\mathrm{ns}}$ \\
\hline Milho/Feijão (PC) & $6,3 \mathrm{C}^{\mathrm{ns}}$ & $7,2 \mathrm{~A}^{\mathrm{ns}}$ \\
\hline Figo & $8,9 \mathrm{Aa}$ & $6,4 \mathrm{Bb}$ \\
\hline Maracujá & $5,7 \mathrm{D}^{\mathrm{ns}}$ & $6,3 \mathrm{~B}^{\mathrm{ns}}$ \\
\hline SAF & $5,5 \mathrm{Db}$ & $6,0 \mathrm{Ba}$ \\
\hline
\end{tabular}

Médias seguidas de mesma letra maiúscula na coluna não diferem significativamente entre os sistemas de uso do solo para cada estação, pelo teste de Scott-Knott a 5\%. A mesma letra minúscula na linha não difere entre as estações para cada sistema de uso do solo, pelo teste $\mathrm{F}$ a $5 \%$. ${ }^{\text {ns }}$ Não-significativo a $5 \%$ pelo teste $\mathrm{F}$ entre as estações. 
com figo com os maiores teores (Tabela 1). Na área de figo, esse comportamento pode ser devido ao uso de cobertura morta proveniente do corte da grama batatais (Paspalum notatum). Essa prática auxilia na manutenção da umidade do solo e evita o contato direto dos raios solares com o solo, diminuindo a temperatura na camada superficial do solo e, conseqüentemente, a mineralização da MOS. Também se pode destacar o uso da leguminosa siratro (Macroptilium artropurpureum) como planta de cobertura para o solo desde o início do plantio da frutífera (1999) até o ano de 2002. A quantidade de matéria seca de folhas provenientes da deiscência natural da cobertura viva formada pelo siratro chegou a $2,0 \mathrm{Mg} \mathrm{ha}^{-1}$, acarretando um estoque de $50 \mathrm{~kg} \mathrm{ha}^{-1}$ de $\mathrm{N}$ provenientes do resíduo dessas folhas (ALMEIDA \& GUERRA, 2008).

Já na área com beringela/milho é utilizada rotação de culturas em sistema plantio direto (SPD), o que permite a manutenção e o acúmulo de resíduos vegetais na superfície do solo. Nessa área também se fez uso de adubação verde com leguminosas em cultivos anteriores, destacando-se mucuna cinza (Mucuna pruriens), mucuna anã (Mucuna deeringiana), Crotalaria spectabilis e Crotalaria juncea, consorciadas com a cultura principal.

De acordo com DUDA et al. (2003), que avaliaram o efeito de diferentes leguminosas (Arachis pintoi, Pueraria phaseoloides e Macroptilium atropurpurem) nos teores de COT também no SIPA e sob Argissolo Vermelho-Amarelo, verificaram que o uso de adubos verdes pode aumentar os teores de COT quando estes são introduzidos junto ao cultivo. Os autores observaram que a manutenção dos resíduos das leguminosas após cada corte promoveu aumentos nos teores COT, enfatizando a importância da utilização dessa prática para melhorar a fertilidade do solo.

Em relação à estação do inverno, a área de beringela/milho $(0-5 \mathrm{~cm})$ apresentou maior teor de COT. Na profundidade de $5-10 \mathrm{~cm}$, nas áreas de milho/feijão e beringela/milho, verificaram-se maiores teores de COT (Tabela 1). Esse comportamento pode ser explicado pela ausência de cobertura morta no figo e pela adoção do SPD na área de beringela/milho, uma vez que no momento da coleta de solo a cultura do milho já havia sido colhida, deixando seus resíduos em superfície para novo plantio da beringela sobre a palhada do milho (0$5 \mathrm{~cm})$. Na área de milho/feijão, o maior teor de COT está relacionado com o revolvimento do solo.

$\mathrm{Na}$ profundidade de $5-10 \mathrm{~cm}$, foram encontradas maiores variações nos teores de COT entre as áreas analisadas no verão. Esse comportamento difere do observado na profundidade de $0-5 \mathrm{~cm}$, onde a adubação orgânica utilizada nas áreas, provavelmente, homogeneizou os teores de COT na camada superficial, como notado nas áreas de milho/ feijão, maracujá e SAF, que apresentaram teores de COT iguais nas duas estações (Tabela 1).

Os sistemas de uso do solo, em cada área, propiciam diferentes quantidades de resíduos vegetais oriundos de cada cultura. As áreas que apresentam menores quantidades desses resíduos, associadas à estação do verão (período com maior disponibilidade de água e temperaturas mais altas), podem ter um aumento em sua decomposição e também na velocidade das reações químicas no solo, com uma mineralização rápida da MOS, conduzindo a teores de COT diferentes em cada área, como observado na tabela 1. Nesta verifica-se que a área de SAF apresentou o menor teor de COT, sendo essa área a mais recente (cinco anos de implantação) e a única em que não se fez uso de nenhum tipo de adubação verde e orgânica em cobertura. Em contrapartida, os sistemas de uso do solo com figo e beringela/milho, onde se fez uso de cobertura morta e de plantio direto respectivamente e também de leguminosas, apresentaram maiores teores de COT e respectivos estoques de COT.

$\mathrm{Na}$ estação do inverno, os sistemas manejados com rotação de culturas (PD e PC) apresentaram os maiores estoques de COT, não sendo observada diferença entre eles (Tabela 1). Esse resultado pode ser decorrente de menores temperaturas nessa estação, fazendo com que se tenha menor mineralização da MOS, principalmente na área com PC, onde os resíduos culturais são adicionados ao solo por meio das práticas agrícolas de aração e gradagem.

A área de beringela/milho apresentou o maior valor de COp na estação do verão, sendo igual ao valor de COp da área do SAF no inverno (Tabela 2). Esse comportamento deve-se ao fato de que essa área é manejada sob SPD e com rotação de culturas. Essa rotação é a mesma da área com milho/feijão, entretanto o PC do solo que é realizado nessa área está propiciando redução nos teores $\mathrm{COp}$ em superfície e, conseqüentemente, menores valores de MOS nessa área. Já para a profundidade de $5-10 \mathrm{~cm}$, foram observados, na área de milho/feijão, valores de COp superiores aos das demais áreas no verão e iguais aos da área de SAF e figo no inverno, pois a fração leve (resíduos culturais) é incorporada ao solo por meio das formas de preparo dessa área.

O aumento dos valores de COp na área do SAF, na estação do inverno, em ambas as profundidades, provavelmente deve-se à incorporação de resíduos culturais advindos de uma poda realizada no próprio sistema, pois, no momento da coleta das amostras para análise (no inverno), o solo estava 
Tabela 2 - Concentração de carbono das frações granulométricas $\left(\mathrm{g} \mathrm{kg}^{-1}\right)$ em função dos diferentes sistemas de uso do solo.

\begin{tabular}{|c|c|c|c|c|}
\hline \multirow[b]{2}{*}{ Sistemas de uso do solo } & \multicolumn{2}{|c|}{------------------COp------------------ } & \multicolumn{2}{|c|}{----------------COam----------------' } \\
\hline & Verão & Inverno & Verão & Inverno \\
\hline Beringela/Milho (PD) & $5,93 \mathrm{Aa}$ & $5,29 \mathrm{Ab}$ & $5,04 \mathrm{D}^{\mathrm{ns}}$ & $4,53 \mathrm{~B}^{\mathrm{ns}}$ \\
\hline Milho/Feijão (PC) & $4,72 \mathrm{Ca}$ & $3,67 \mathrm{Cb}$ & $4,76 \mathrm{Db}$ & $5,46 \mathrm{Aa}$ \\
\hline Figo & $5,30 \mathrm{Ba}$ & $4,52 \mathrm{Bb}$ & $8,40 \mathrm{Aa}$ & $4,54 \mathrm{Bb}$ \\
\hline Maracujá & $3,52 \mathrm{D}^{\mathrm{ns}}$ & $3,30 \mathrm{D}^{\mathrm{ns}}$ & $5,55 \mathrm{C}^{\mathrm{ns}}$ & $5,47 \mathrm{~A}^{\mathrm{ns}}$ \\
\hline SAF & $2,83 \mathrm{~Eb}$ & $5,45 \mathrm{Aa}$ & $6,61 \mathrm{Ba}$ & $3,48 \mathrm{Cb}$ \\
\hline Beringela/Milho (PD) & $2,64 \mathrm{Ba}$ & $2,15 \mathrm{Bb}$ & $6,89 \mathrm{~B}^{\mathrm{ns}}$ & $7,17 \mathrm{~A}^{\mathrm{ns}}$ \\
\hline Milho/Feijão (PC) & $3,08 \mathrm{~A}^{\mathrm{ns}}$ & $3,19 \mathrm{~A}^{\mathrm{ns}}$ & $5,80 \mathrm{Cb}$ & $7,15 \mathrm{Aa}$ \\
\hline Figo & $2,23 \mathrm{Bb}$ & 3,45 Aa & $9,27 \mathrm{Aa}$ & $4,38 \mathrm{Db}$ \\
\hline Maracujá & $1,47 \mathrm{C}^{\mathrm{ns}}$ & $1,80 \mathrm{~B}^{\mathrm{ns}}$ & $5,95 C^{\mathrm{ns}}$ & $6,40 \mathrm{~B}^{\mathrm{ns}}$ \\
\hline SAF & $1,46 \mathrm{Cb}$ & $2,91 \mathrm{Aa}$ & $5,58 C^{\mathrm{ns}}$ & $5,15 \mathrm{C}^{\mathrm{ns}}$ \\
\hline
\end{tabular}

Médias seguidas de mesma letra maiúscula na coluna não diferem significativamente entre os sistemas de uso do solo para cada estação, pelo teste de Scott-Knott a 5\%. A mesma letra minúscula na linha não difere entre as estações para cada sistema de uso do solo, pelo teste F a 5\%. ${ }^{n s}$ Não-significativo a $5 \%$ pelo teste $\mathrm{F}$ entre as estações. COp=carbono orgânico particulado; COam=carbono orgânico associado aos minerais.

recoberto com resíduos vegetais, diferente da época de coleta da estação do verão, o que refletiu em menores valores de COp nesta área (Tabela 2).

Verificou-se que o COp mostrou-se eficiente para evidenciar diferenças entre as áreas analisadas, principalmente na profundidade de $0-5 \mathrm{~cm}$ (Tabela 2), no verão, quando ocorre o maior acúmulo dos resíduos vegetais e a influência direta do manejo e da rotação/ consorciação de culturas. Avaliando a influência do aumento da intensidade de utilização das pastagens de inverno e diferentes sistemas de culturas de verão sobre a dinâmica da MOS e de suas frações granulométricas de um Argissolo Vermelho-Amarelo em áreas de integração lavoura-pecuária sob SPD, NICOLOSO (2005) constatou que o COp foi mais eficaz para avaliar as modificações nos teores de carbono orgânico decorrentes do manejo, em especial nos primeiros centímetros do solo $(0,0-2,5$ e $2,5-5,0 \mathrm{~cm})$.

Os resultados obtidos por Nicoloso (2005) e também os encontrados neste estudo demonstram que a variação dos teores de COp entre as diferentes áreas estudadas, na profundidade de $0-5 \mathrm{~cm}$, é dependente da adição de resíduos vegetais, ou seja, sistemas de uso do solo que propiciem a adição desses resíduos na superfície do solo influenciam a manutenção dos valores de COp.

Por meio das diferenças observadas na tabela 2, verificou-se que, na fração COp, as mudanças provenientes dos diferentes sistemas de uso do solo são mais sensíveis, em comparação com o COT na camada superficial do solo, pois este não apresentou diferenças entre todas as áreas analisadas nessa profundidade (Tabela 1). Desse modo, verifica-se que o COp pode funcionar como indicador da qualidade da MOS em relação às alterações de manejo e a curto prazo. Esse comportamento também foi observado por CONCEIÇÃO et al. (2005), avaliando a qualidade do solo sob diferentes sistemas de manejo em experimentos de longa duração no Sul do Brasil com cereais e leguminosas, na profundidade de $0-5 \mathrm{~cm}$.

O COam é, normalmente, menos modificado pelas diferentes formas de manejo adotadas, principalmente a curto prazo (BAYER et al., 2004). Entretanto, verificaram-se diferenças entre as áreas nas duas profundidades avaliadas, sendo observados maiores valores para as áreas de figo e SAF $(0-5 \mathrm{~cm})$ e figo e beringela/milho $(5-10 \mathrm{~cm})$ no verão. No inverno, as áreas de milho/feijão e maracujá $(0-5 \mathrm{~cm})$ e beringela/ milho e milho/feijão $(5-10 \mathrm{~cm})$ apresentaram os maiores teores de COam (Tabela 2). Essas diferenças podem estar associadas às práticas agroecológicas utilizadas, como adubação verde, rotação e/ou consorciação de culturas, adubação orgânica e manutenção dos resíduos vegetais em superfície, com exceção à área de milho/feijão (PC). Essas práticas utilizadas com freqüência, associadas ao histórico e tempo de uso de cada área, podem estar influenciando as diferenças encontradas, pois a textura do solo é a mesma em todas as áreas.

Na camada de $0-5 \mathrm{~cm}$, os valores de COp, na estação do inverno, foram mais apropriados para identificar as mudanças provenientes dos sistemas de 
uso do solo do que os valores de COam. Entretanto, na profundidade de $5-10 \mathrm{~cm}$, estação do inverno, esse comportamento foi observado para o COam. Esse resultado pode ser devido a uma menor influência dos resíduos vegetais nessa profundidade $(5-10 \mathrm{~cm})$ e uma maior atuação da matéria orgânica na formação de microagregados, já que os maiores valores de COam foram observados nas coberturas com maiores teores de COT (Tabelas 1 e 2). Segundo NICOLOSO (2005), os estoques de COT são compostos em mais de $80 \%$ pelo COam.

\section{CONCLUSÕES}

Os sistemas de uso do solo com figo e beringela/milho (PD) são mais eficientes em acumular carbono que os demais, pois apresentam maiores estoques de COT no verão. Na estação do inverno, esse comportamento foi observado para os sistemas com rotação de culturas (PD e PC), não sendo verificada diferença entre estes.

O COp mostrou-se mais adequado que o COT para evidenciar diferenças provenientes do efeito dos sistemas de uso do solo na profundidade de 0$5 \mathrm{~cm}$, na estação do verão. As diferenças observadas entre os sistemas de manejo para o COam podem ser decorrentes do uso constante da adubação orgânica, associadas ao histórico e tempo de uso de cada área.

\section{REFERÊNCIAS}

ALMEIDA, D.L.; GUERRA, J.G.M. Uma experiência de pesquisa em agricultura orgânica: “fazendinha agroecológica- km 47”. Acesso: 20 mar. 2008. Disponível em: http://www.pronaf.gov.br/ dater/arquivos/ 27_Experiencia_em_Pesquisa_Agric_Org.pdf.

BAYER, C. et al. Armazenamento de carbono em frações lábeis da matéria orgânica de um Latossolo Vermelho sob plantio direto. Pesquisa Agropecuária Brasileira, Brasília, v.39, n.7, p.677-683, 2004. Disponível em: <http://www.scielo.br/scielo.php?pid=S0100204X2004000700009\&script=sci_arttext\&tlng=en $>$. Doi: 10.1590/ S0100-204X2004000700009.
CAMBARDElla, C.A.; ELliOTT, E.T. Particulate soil organic-matter changes across a grassland cultivation sequence. Soil Science Society of America Journal, Madison, v.56, p.777-783, 1992.

CARDOZO, S.V. et al. Caracterização de propriedades edáficas em áreas sob manejo orgânico e natural na região serrana do Estado do Rio de Janeiro. Semina. Ciências Agrárias, Londrina, v.29, p.517-530, 2008.

CHRISTENSEN, B.T. Structure and organic matter storage in agricultural soils. In: CARTER, M.R.; STEWART, B.A. (Ed.). Structure and organic matter storage in agricultural soils. Boca Raton: CRC, 1996b. p.97-165.

CONCEIÇÃO, P.C. et al. Qualidade do solo em sistemas de manejo avaliada pela dinâmica da matéria orgânica e atributos relacionados. Revista Brasileira de Ciência do Solo, Viçosa, v.29, n.5, p.777-788, 2005. Disponível em: <http:// w w w. s c i e 1 o.br/s c i e 1 o.ph p ? p id = S $0100-$ $06832005000500013 \&$ script $=$ sci_arttext $>$. Doi: $10.1590 /$ S0100-06832005000500013.

DUDA, G.P. et al. Perennial herbaceous legumes as live soil mulches and their effects on $\mathrm{C}, \mathrm{N}$ and $\mathrm{P}$ of the microbial biomass. Scientia Agricola, Piracicaba, v.60, p.139-147, 2003. Disponível em: <http://www.scielo.br/scielo.php?pid=S0103$90162003000100021 \&$ script $=$ sci arttext $>$. Doi: 10.1590/ S0103-90162003000100021.

EMBRAPA - Empresa Brasileira de Pesquisa Agropecuária.Centro Nacional de Pesquisa de Solos. Sistema brasileiro de classificação de solos. Brasília: Embrapa Produção de Informação, 2006. 306p.

EMBRAPA. Centro Nacional de Pesquisa de Solos. Manual de métodos de análise de solo. 2.ed. Rio de Janeiro, 1997. 212 p.

GOLCHIN, A. et al. Soil structure and carbon cycling. Australian Journal of Soil Research, Victoria, v.32, p.10431068, 1994a.

NICOLOSO, R.S. Dinâmica da matéria orgânica do solo em áreas de integração lavoura-pecuaria sob sistema de plantio direto. 2005. 150f. Dissertação (Mestrado em Agronomia) - Programa de Pós-graduação em Ciência do Solo, Centro de Ciências Rurais, Universidade Federal de Santa Maria. 